\title{
Comparison of Preregistration Platforms
}

\author{
Steve Haroz - Inria \& Universite Paris-Saclay
}

\section{Abstract}

Preregistration forces researchers to front-load a lot of decision-making to an early stage of a project. Choosing which preregistration platform to use is one of those early decisions, and because a preregistration cannot be moved, that choice is permanent. This article aims to help researchers who are already interested in preregistration choose a platform by clarifying differences between them. Preregistration criteria and features are explained and analyzed for popular sites that cater to a broad range of research fields, including: GitHub, AsPredicted, Zenodo, the Open Science Framework (OSF), and an "open-ended" variant of OSF. Platforms such as ClinicalTrials.gov for clinical research and PROSPERO for systematic reviews are not compared because they are limited to specific types of studies rather than general purpose research. While a private prespecification document can help mitigate self-deception, this guide considers publicly shared preregistrations that aim to improve credibility. It therefore defines three of the criteria (a timestamp, a registry, and persistence) as a bare minimum to meet the definition of a preregistration. Additional helpful features are also listed. GitHub and AsPredicted do not meet all three basic criteria. Zenodo and OSF meet the basic criteria and vary in which additional features they offer.

\section{What is a Preregistration?}

A preregistration is a prespecification of a data collection and analysis plan that has been registered on a public repository, so readers can compare it to the report of a study actually conducted. It facilitates differentiating post-hoc data collection and analysis used to generate hypotheses from a prespecified study used to test hypotheses.

More information about preregistration and its rationale, an overview, a collection of more in-depth resources, and answers to frequently asked questions are available at https://cos.io/initiatives/prereg.

Table 1. The features of general-purpose preregistration services. A text description is given for features that cannot be described with a simple positive or negative score.

Flexibility - Limited: Only limited deviation from a template is possible. External: An external tool allows unconstrained editing. Collaboration - Approval: Filing can await collaborator approval, but only the primary author can edit. Sequential: Collaborators can edit, but not at the same time. External: Editing is done via an external tool.

Usability - Difficulty for novice users ranging from very easy to easy to moderate to skill needed.
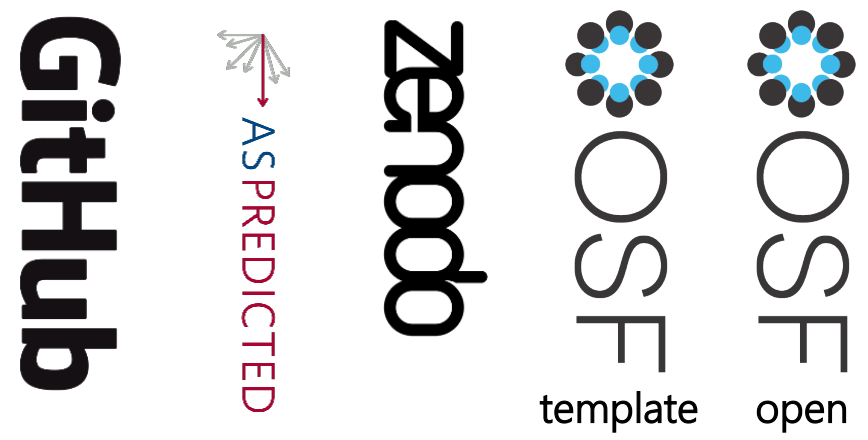

\begin{tabular}{|c|c|c|c|c|c|}
\hline$\star$ Timestamp & $x$ & $\checkmark$ & $\checkmark$ & $\checkmark$ & $\checkmark$ \\
\hline$\star$ Indexed Registry & $x$ & $x$ & $\checkmark$ & $\checkmark$ & $\checkmark$ \\
\hline$\star$ Persistence & $x$ & $\checkmark$ & $\checkmark$ & $\checkmark$ & $\checkmark$ \\
\hline Anonymity & $x$ & $\checkmark$ & $x$ & $\checkmark$ & $\checkmark$ \\
\hline Additional Materials & $\checkmark$ & $x$ & $\checkmark$ & $\checkmark$ & $\checkmark$ \\
\hline Sandbox & $\checkmark$ & $\checkmark$ & $\checkmark$ & $\checkmark$ & $\checkmark$ \\
\hline Template & $x$ & $\checkmark$ & $x$ & $\checkmark$ & $x$ \\
\hline Rich Formatting & $\checkmark$ & $x$ & $\checkmark$ & $x$ & $\checkmark$ \\
\hline Tracked Changes & $\checkmark$ & $x$ & $x$ & $\checkmark$ & $x$ \\
\hline Flexibility & external & limited & external & limited & external \\
\hline Collaboration & external & approval & external & sequential & external \\
\hline Usability & skill needed & very easy & moderate & easy & moderate \\
\hline
\end{tabular}




\section{Criteria}

1. Timestamp: It is critical that a preregistration is immutable with a timestamp to verify that it was not altered after the data was analyzed or the manuscript submitted. This criterion is the pre part of preregistration. Without an immutable timestamp, a platform is not appropriate for preregistration.

2. Indexed Registry: Registration is the second critical part of preregistration. It needs to eventually be indexed and searchable. To understand why, examine how some twitter accounts seem to make prophetic predictions about sporting or political outcomes. There is documented evidence (archived here) of accounts tweeting dozens of predictions and deleting any that do not come true. But the deletions do not show up in the account's timeline. So the account seems to only make precise and highly unexpected predictions that come true later. However, if the tweets were indexed, you could detect that the account deleted many tweets on that day, even if you could not read them. A registry that indexes all preregistrations browsable by account protects from that kind of hacking. Even if a preregistration were deleted, the author list and a note would be left behind. Without indexed registration, a platform is prone to this kind of "preregistration hacking".

3. Persistence: A preregistration will be referenced from the eventual writeup, so persistence of the content and of an immutable unique identifier - such as a DOI - is critical to ensure that it does not suffer from "link rot". Otherwise, a preregistration may be not visible to future readers of the publication.

4. Anonymity: Is it possible to use this preregistration platform for venues that require an anonymous submission? This feature necessitates creating a view of a preregistration that omits any name or affiliation fields. Lacking this feature could prevent the submission of preregistered work to some venues.

5. Additional materials: Can you include analysis scripts, experiment materials, or pilot data along with the preregistration? Including an analysis as part of a preregistration can be especially helpful in showing that all of the detailed decisions were determined a priori.

6. Sandbox: Is there a "sandbox" mode where you can try out the process and features without it being indexed or leaving a permanent record? Preregistering for the first time can be daunting, so being able to practice without consequences may reduce barriers for some. Also, for anyone teaching it in a course, it's important to not create a useless preregistration every time it is demonstrated in class.

7. Template: Does the site have one or more templates to choose from or that it links to? Or do you need to seek out what information to include in a preregistration? Even for those with experience preregistering, it can be easy to accidentally leave off important information without a template.

8. Rich Formatting: Can styled text, bulleted lists, and figures be used in the preregistration? These features can help clarify and separate definitions, thresholds, intermediate constructs, and multiple exclusion criteria or statistical tests. More complex analyses may benefit from being broken down with bulleted or numbered lists. Experiment conditions or visual stimuli may be best explained using a diagram (see Fig. 1). Also, what are the options for writing tools? Using an external program such as Microsoft Word or Google Docs may be desirable for spelling and grammar checks and for text formatting.

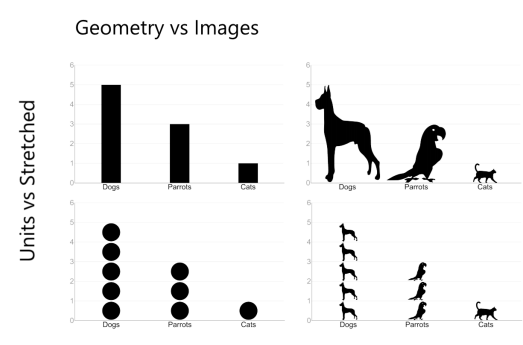

Figure 1. This example of stimuli variations may not be conveyed clearly and succinctly with text, so a figure would help simplify explaining the conditions in a preregistration. Used with permission under CC-BY license.

9. Tracked changes: If the preregistration is updated or a deviation needs to be documented, is it clear that an update occurred? Can the changes be documented? Is there some sort of "diff" to show what changed?

10. Flexibility: If there is a template, can you skip a question, add a question, or otherwise deviate from the specified fields? Not every field may be applicable to every project. Note that flexibility has benefits (e.g., using a different template) and downsides (e.g. it is easier for people to omit critical information), so a text description is given rather than a positive or negative score.

11. Collaboration: On projects with multiple authors, can everyone edit or proofread the preregistration before it is filed? This issue can be especially important when not everyone on the project has the same level of experience or access to the same information.

12. Usability: Quality of life and avoiding frustration for novices and experts are important.

\section{GitHub}

GitHub (http://github.com) is a version control system that is primarily intended for software development. Although it is 
not touted as a preregistration platform, it has been used as one. It should not be.

\section{Timestamp: $X$}

It is easy to fake a timestamp on GitHub. Set your computer's time back. Make a repository. Commit something. Then reset your computer's time and push the repository to GitHub. Because GitHub has no "uploaded" timestamp, there's no way to tell when the preregistration was actually posted. Before data collection? Or right after? It would be easy seem psychic by recording winners from every sports game in the past 70 years, and give the commit a date from 1955. Unless GitHub changes how it records timestamps, it can be manipulated.

\section{Indexed Registry: $X$}

You can make a repository private, delete it, or give it a different URL without any trace that such a change occurred. Worse, you can delete a repository then upload a new one to the same URL, which could allow a nefarious author to change information after data collection or publication without any indication that the change occurred.

\section{Persistence: $X$}

While GitHub is likely to persist for a very long time, there is no written statement that content on the service will continue to be hosted publicly, especially for free accounts. GitHub can choose to delete all of an account's content or make it private. Whether or not the content is mentioned in a publication is irrelevant.

\section{Anonymity: $\mathrm{X}$}

It is not possible to hide the owner of a GitHub repository or its commit authors unless a separate anonymous GitHub account is created for each user of a repository.

\section{Additional materials:}

Any files can be included in the repository.

\section{Sandbox:}

You can create a private repository which will not be indexed. And any repository can be deleted or have its history changed. GitHub is its own sandbox.

\section{Template: $\mathrm{X}$}

GitHub is a general-purpose repository, so it has no templates or other information about preregistration.

\section{Rich formatting:}

GitHub has a built-in markdown editor for rich text. Alternatively, any arbitrary file, like a PDF or Microsoft Word document, can be uploaded. Consequently, you can choose your own authoring tools. GitHub has a built-in ability to display formatted markdown or PDFs.

\section{Tracked changes:}

Git commits are all available, so any version can be viewed. And if a document format enables comparison, a diff of the changes can be inspected.

\section{Flexibility: High.}

GitHub has a built-in markdown editor. Alternatively, any arbitrary file, like a PDF or Microsoft Word document, can be uploaded. Consequently, you can choose your own authoring tools. GitHub has a built-in ability to display formatted markdown or PDFs.

\section{Collaboration: Sequential editing.}

Collaboration with GitHub is certainly possible, but it doesn't happen in real time. Each user must "push" edits and "pull" updates to synchronize with co-authors. Also, seeing changes (diffs) only works well for simple text documents. More complex documents like a Microsoft Word document are not wellsupported.

\section{Usability: Training needed.}

GitHub requires some education or experience with source control, but that skill has general purpose uses for contributing to software development in general. For those without experience in source control, user interfaces like GitHub Desktop and SourceTree substantially simplify the process.

\section{Overview}

A GitHub repository does not provide an immutable timestamp nor an indexed record. So authors should avoid using GitHub for preregistration. Reviewers and readers should generally be very skeptical of articles that claim to have preregistered on GitHub. However, see the hybrid option below.

\section{AsPredicted}

AsPredicted (http://aspredicted.org) is singularly a preregistration platform rather than a general-purpose platform that happens to enable preregistration. It is popular for its simplicity and ease of use.

\section{Timestamp:}

AsPredicted includes an immutable timestamp when the form is submitted.

\section{Indexed Registry: X}

AsPredicted does not require preregistrations to ever become public. So one could "preregistration hack" by creating multiple preregistrations but only publicizing the most favorable.

3. Persistence: $\checkmark$

Preregistrations are stored on the Web Archive once they are made public. 


\section{Anonymity:}

A "blind" URL is available that removes author information.

\section{Additional materials: $X$}

AsPredicted preregistrations consist of only form text.

\section{Sandbox:}

There is a "Just Testing" checkbox on the form which will (1) prevent the preregistration from being indexed and (2) delete it after 24 hours.

\section{Template:}

AsPredicted has one template.

\section{Rich formatting: $X$}

It is a web form with radio buttons and plain text boxes. Formatting text is not possible.

\section{Tracked changes: $X$}

AsPredicted does not have this feature.

\section{Flexibility: Very limited.}

Some questions can be left unanswered. It is not possible to add other sections or questions, but there is an "other" text box for miscellaneous information.

\section{Collaboration: Only for approval.}

Only one person can edit the preregistration. But all authors must approve it before it is filed.

\section{Usability: Very easy.}

AsPredicted is by far the simplest to use. It consists of a few textboxes and radio buttons. There is even a "just testing" option that deletes the preregistration after 24 hours.

\section{Overview}

The simplicity of AsPredicted can facilitate learning how to think about preregistration for those who have not done it before. However, the lack of a searchable index calls into question whether it actually is a preregistration or simply a timestamped file.

\section{Zenodo}

Zenodo (http://zenodo.org) is a platform for archiving and registering scientific materials. It is not commonly used for preregistration, and it does not even have a preregistration category. But uploading a document tagged with the "other" category can serve as a preregistration.

\section{Timestamp:}

The timestamp is oddly difficult to find. A prominently visible publication date is not relevant because it can be set to any arbitrary date. There is, however, an upload timestamp that is not visible in the user interface. On the side bar is a collection of options under "Export". Selecting "JSON" encodes all of the meta-data including "created" and "updated" timestamp fields around 40-50 lines down from the bottom. While it is technically present, it is not very discoverable.

\section{Indexed Registry:}

The upload is given a DOI and is indexed and searchable.

\section{Persistence: $\checkmark$}

Zenodo is run by CERN and OpenAIR and states the following in its FAQ: "Both data files and metadata are kept in multiple online and independent replicas. CERN has considerable knowledge and experience in building and operating large scale digital repositories and a commitment to maintain this data centre to collect and store 100s of PBs of LHC data as it grows over the next 20 years. In the highly unlikely event that Zenodo will have to close operations, we guarantee that we will migrate all content to other suitable repositories, and since all uploads have DOIs, all citations and links to Zenodo resources (such as your data) will not be affected." https://help.zenodo.org

\section{Anonymity: $X$}

There does not appear to be any way of hiding author information on Zenodo.

\section{Additional materials:}

Zenodo allows multiple files to be uploaded. Because the website's user interface does provide a way to make directories, any new category of material (i.e. analysis code, data, or experiment code) would need to be uploaded as a ZIP file or posted into a separate upload.

\section{Sandbox:}

http://sandbox.zenodo.org is an unindexed version of the site whose content is not visible on the main site.

\section{Template: $X$}

Zenodo is a general-purpose open science repository, so it has no templates or other guidance about preregistration. However, you can copy another site's preregistration form and upload that document to Zenodo.

\section{Rich formatting:}

Zenodo has no built-in document editor, so editing is as flexible as the user's word processor of choice.

\section{Tracked changes: $X$}

Zenodo has versioning and shows a message if an updated version exists. But it does not show what changed between versions.

\section{Flexibility: External editor.}

Because there is no built-in template, Zenodo relies on the user's external editor. 


\section{Collaboration: Via external tools.}

Because Zenodo's content is uploaded, collaboration can be done via whatever application is used to create the file. So if a preregistration is made in Google Docs or Microsoft Word, collaboration is done within those applications. There is unfortunately no mechanism for coauthors to approve the metadata or upload on Zenodo. However, Zenodo does allow metadata to be edited after the fact in case a co-author wants changes.

\section{Usability: Moderate.}

Zenodo's interface is clean and straight forward to use. It is easy to upload material and provide basic metadata. However, the lack of guidance around preregistration may cause difficulty for beginners and may require using another site to find a template. Moreover, the upload date seems needlessly difficult to find for readers.

\section{Overview}

Zenodo has all of the basic features, and its flexibility allows for types of preregistration not supported by standard templates. Nevertheless, preregistration is not well supported by the UI, providing no links to or mentions of any templates. This lack of any preregistration guidance may be a challenge for novices and an annoyance for any user who just wants to use a standard template.

\section{Open Science Framework (template)}

Besides being a general-purpose open science platform, OSF (http://osf.io) has a portal specifically for preregistrations. The site uses the more general term "registration" because the site allows even a completed project to be registered to create an immutable milestone. The registration can optionally be connected to project files and preprint. Consequently, data collection and analysis material can be included in the preregistration, and data collected later and even the preprint can be connected, which can help keep all the components of a project in one place.

\section{Timestamp:}

OSF includes an immutable timestamp of when the preregistration was filed.

\section{Indexed Registry:}

The preregistration is given a unique identifier and a DOI can optionally be added. All public registrations are indexed and searchable, and registrations can only be private for a limited embargo period of a few years at most. The finite embargo inhibits a nefarious person from filing many different variations of a preregistration and only making the one with the best results public.

\section{Persistence:}

OSF is run by the Center for Open Science (COS), and OSF states the following in its FAQ:

"COS established a $\$ 250,000$ preservation fund for hosted data in the event that COS had to curtail or close its offices. If activated, the preservation fund will preserve and maintain read access to hosted data. This fund is sufficient for $50+$ years of read access hosting at present costs. COS will incorporate growth of the preservation fund as part of its funding model as data storage scales."

-https://help.osf.io/hc/en-us/articles/360019737894-FAQs

The site also collaborates with the Internet Archive (http://archive.org) for ensuring long term preservation. See https://www.cos.io/about/news/center-open-science-and-internet-archive-announce-collaboration-preserve-open-data

\section{Anonymity:}

Authors can create a special URL for a private project that makes the project visible but with identifying information removed from the metadata. In cases were anonymous submission is needed, the preregistration will not give away the identity of the authors. However, any information in the content or documents will not be affected, so it is important to avoid including names in the forms.

\section{Additional materials:}

Because a preregistration is just a special case of a project registration, all the files from a project are included. Consequently, data collection, analysis code, and pilot results can be included and accessed from the preregistration. However, the preregistration interface does not clearly indicate whether or what types of files are present, so it's important to indicate in the text of the form what information is in the files.

\section{Sandbox:}

http://test.osf.io is a sandbox version of the site. Its projects, registrations, and even user accounts are completely isolated from the main site and are not visible from search engines. A new account needs to be made for the test site. It is important to remember that the test site may erase all information at any time, so avoid relying on it for anything more than testing features or getting practice with preregistration.

\section{Template:}

OSF has a collection of templates for quantitative and qualitative research.

\section{Rich Formatting: $X$}

It is a web form with radio buttons and plain text boxes. Formatting text is not possible. While images can be included in the supplemental files, they cannot appear inline in the preregistration, and there is not a clear way to link to them.

\section{Tracked changes:}


Versioned updates can be made to a preregistration. The changes are highlighted in the new version, and an explanation for the changes is shown.

\section{Flexibility: Limited.}

While there is some flexibility in which template can be chosen, and not all questions are mandatory, there is very limited flexibility in how the templates can be filled in.

\section{Collaboration: Sequential editing and approval.}

OSF allows others with OSF accounts to be invited as contributors to a preregistration. All contributors can view in-progress registrations under their drafts and can edit them. The editing is not instantaneous like a Google Doc, so simultaneous editing will not work. However, sequential editing and proofreading among all contributors is straight forward. Once it is filed, all contributors have the option of approving or rejecting the preregistration. If 48 hours pass, the preregistration will be approved even without all contributors approving it to avoid a project being blocked from an inactive contributor.

\section{Usability: Easy.}

The form and process of filing the preregistration are simple with OSF's preregistration site that allows for saving as the user progresses and allows another collaborate to pick up where another left off. However, a common question that new users struggle with is "Which preregistration template do I choose?" In many ways, having different options can be beneficial. However, confusing some new users is a consequence.

\section{Overview}

OSF is the only platform with built-in preregistration templates that meets the three core criteria of a preregistration (i.e. a timestamp, a registry, and a persistence plan). There is some limited flexibility in filling in the template form, however, the open-ended option is available, which trades template guidance for flexibility.

\section{Open Science Framework (open-ended)}

One of the templates available on OSF (https://osf.io) is the "Open-Ended Registration", which is sufficiently different from the other templates that it warrants its own category. The form only asks for a title, description, and summary. Like the other templates, it allows for uploading files, and it will archive the files in a connected OSF project. The open-ended registration allows for total flexibility in both templates and authoring tools. The downside is that like Zenodo and Github, it requires the extra steps of finding a template to copy. However, as OSF has other templates on the same site, it is simple to start another draft registration on OSF and copy the questions from that draft to your own document.
Two main benefits of the open template are (a) the ability to use any template and (b) the ability to use all features of a word processor such as Google Docs, including real-time collaborative editing, comments, rich formatting, and images.

\section{Timestamp:}

OSF includes an immutable timestamp of when the preregistration was filed.

\section{Indexed Registry:}

The preregistration is given a unique identifier and a $\mathrm{DOI}$ can optionally be added. All public registrations are indexed and searchable, and registrations can only be private for a limited embargo period of a few years at most. The finite embargo inhibits a nefarious person from filing many different variations of a preregistration and only making the one with the best results public.

\section{Persistence: $\checkmark$}

OSF is run by the Center for Open Science (COS), and OSF states the following in its FAQ:

"COS established a $\$ 250,000$ preservation fund for hosted data in the event that COS had to curtail or close its offices. If activated, the preservation fund will preserve and maintain read access to hosted data. This fund is sufficient for $50+$ years of read access hosting at present costs. COS will incorporate growth of the preservation fund as part of its funding model as data storage scales."

-https://help.osf.io/hc/en-us/articles/360019737894-FAQs

The site also collaborates with the Internet Archive (http://archive.org) for ensuring long term preservation. See https://www.cos.io/about/news/center-open-science-and-internet-archive-announce-collaboration-preserve-open-data

\section{Anonymity:}

Authors can create a special URL for a private project that makes the project visible but with identifying information removed from the metadata. In cases were anonymous submission is needed, the preregistration will not give away the identity of the authors. However, any information in the content or documents will not be affected, so it is important to avoid including names in the forms.

\section{Additional materials:}

Because a preregistration is just a special case of a project registration, all the files from a project are included. Consequently, data collection, analysis code, and pilot results can be included and accessed from the preregistration. However, the preregistration interface does not clearly indicate whether or what types of files are present, so it's important to indicate in the text of the form what information is in the files. 


\section{Sandbox:}

http://test.osf.io is a sandbox version of the site. Its projects, registrations, and even user accounts are completely isolated from the main site and are not visible from search engines. A new account needs to be made for the test site. It is important to remember that the test site may erase all information at any time, so avoid relying on it for anything more than testing features or getting practice with preregistration.

\section{Template: $\mathrm{X}$}

For the open-ended preregistration, you make your own document. However, you can make a draft registration using one of the other OSF templates and copy the questions to your document.

\section{Rich Formatting: $\checkmark$}

With the open-ended template and uploaded file, you are using an external editor, which is likely a full-featured word processor.

\section{Tracked changes: $X$}

The ability to show what updated and why on OSF preregistrations does not extend to files.

\section{Flexibility: External editor.}

Unlike OSF's templates, the flexibility of the external editor allows for adding or skipping template questions or using a template not listed

\section{Collaboration: Via external tools.}

If using a tool like Google Docs, you have the option of realtime collaborative editing and commenting.

\section{Usability: Moderate.}

As can be seen from the instructions, the increased flexibility and rich formatting comes with a slightly more involved process compared with using a built-in template form.

\section{Overview}

There is a tradeoff between simplicity and flexibility when using the built-in OSF template forms compared to using an OSF open-ended registration with an uploaded document. The choice comes down to the importance of for template flexibility and rich text (which the open-ended template allows for) versus the simplicity of the built-in template.

\section{Tutorial}

To create an open-ended template with an externally created preregistration:

1) Make a document in your format of choice. Google Drive documents allow for real-time collaboration with contacts to avoid confusion about versions. However, such as Microsoft Word or a markdown-to-PDF converter are perfectly valid alternatives.
2) Copy the questions from an existing template of your choice.

3) Make an OSF project at https://osf.io

4) Upload the preregistration document

5) Optional: Consider sharing your analysis code, stimuli, and any other replication or reproducibility materials in the OSF project to demonstrate the thoroughness of the prespecification.

6) Pause. Filing a preregistration cannot be undone. While you can file an additional follow-up preregistration if you find an error, it requires awkwardly pointing out the error. Proof read it carefully. Make sure any collaborators have proofread it too. Double check that you uploaded the most recent version of the document to OSF.

7) File the preregistration.

a. Go to "Registrations" in the project view.

b. Click "New Registration".

c. Select "Open-Ended Registration".

d. Fill in the basic details.

e. Select the preregistration file from the OSF project to include.

f. Double check everything!

g. Finalize and post the preregistration.

8) Collect and analyze data.

9) Remember to report the preregistration in your manuscript and explicitly describe any deviations from the initial plan.

\section{Preregistration tips}

1) Eliminate ambiguity. The variables, conditions, statistical tests or measures, and how the results will be interpreted to answer the questions need to be unambiguously interpretable to eliminate as much flexibility as possible.

2) Do not define or specify anything more than once. It is too easy to update one instance and then forget to update the other, resulting in an ambiguous contradiction.

3) Pilot before preregistering. Pilot data doesn't count as existing data as long as the analysis does not include the pilot data.

4) Use pilot data to create the analysis code before preregistering, and include the code with the preregistration.

5) Do not use imprecise language like "we may" or "we might".

6) Keep it short. Try to simplify the content, so reviewers can quickly skim over it and compare it to the manuscript.

7) Never Preregister After Results are Known (Never PARK).

8) Use a staging or test site to try preregistering at least one before doing it for real. See how the information looks and is organized.

9) Ask someone who has preregistered a study previously to proofread yours.

10) Don't fret over deviations. It is not a big problem if you find a problem after collecting the data. Need different 
exclusion criteria? Need to modify your analysis? Just be transparent by clearly reporting it in the manuscript. 